\title{
A Sophisticated Job Scheduling Approach to Minimize the Time Consumption at Computational Cloud
}

\author{
Jyoti Verma \\ M.Tech(SCHOLAR) \\ Punjab
}

\author{
Heena Gulati \\ AP (CSE) \\ RIET, RAILMAJRA, Punjab
}

\author{
Harish Kundra \\ HOD (CSE) RIET,RAILMAJRA \\ RIET, RAILMAJRA, Punjab
}

\begin{abstract}
Cloud Computing is an evolving paradigm of this new world. Time complexities have demanded sophisticated scheduling algorithms form the user to prevent the over burden of the cloud servers. Although the cloud servers are fast in comparison to the other platforms of servers but still a good scheduling algorithm would enhance the performance of the job execution. This paper focuses on the creation of a sophisticated job scheduling approach consisted of time constrain algorithms and grouping based algorithm.
\end{abstract}

\section{Keywords}

Cloud Computing, Job Scheduling, Group Based Scheduling, Time Constraint Scheduling.

\section{INTRODUCTION}

Cloud computing is the use of the Internet for the tasks performed on computer. The "cloud" represents the Internet. Cloud Computing is a Service that stores and retrieve the information. Flickr is the example for cloud computing as service. It is a platform that is the basic structure on which the application stands. Through cloud computing web is becoming the platform. Cloud computing is the process of taking the services and the tasks performed by computers and bringing back to the web. The Security of Cloud Computing Users study shows that when it comes to cloud computing the glass may be half full or half empty because only half or less of respondents have positive perceptions about how their organizations are adopting cloud security best practices and creating confidence in cloud services used within their organization. In a cloud computing environment, the traditional role of service provider is divided into two: the infrastructure providers who manage cloud platforms and lease resources according to a usage-based pricing model, and service providers, who rent resources from one or many infrastructure providers to serve the end users. The emergence of cloud computing has made a tremendous impact on the Information Technology (IT) industry over the past few years, where large companies such as Google, Amazon and Microsoft strive to provide more powerful, reliable and costefficient cloud platforms, and business enterprises seek to reshape their business models to gain benefit from this new paradigm.

\subsection{Cloud based delivery}

Cloud computing has become a Buzz word in the IT Industry. It is composed of several layers which can be accessed by users getting cloud-based services. The following four key layers of a cloud computing environment will help understand the functionality and the technical skills required, in order to understand the system as well as perform tasks more efficiently.

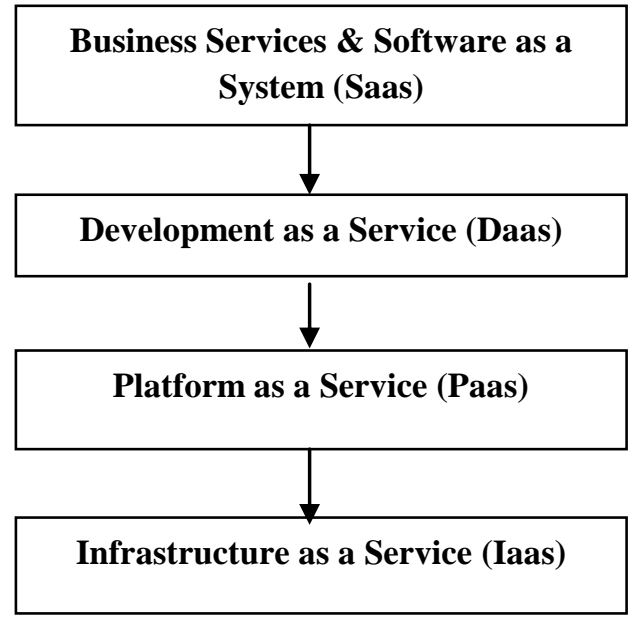

Fig.1: Layers of cloud based delivery

\subsubsection{IAAS}

It is often referred as a visualization layer. It forms the basis of cloud environment. It comes into existence after installing operating systems at virtual machines and allows users to organize large number of virtual machines [3].

\subsubsection{PAAS}

It forms the basis of accessing and connecting multiple devices in the cloud environment and is required to compute any resource utilization or scheduling processes.

\subsubsection{DAAS}

This layer ensures that cloud services have been set up, organized and managed properly with appropriate applications and maintaining optimal performance settings.

\subsubsection{SAAS}

At this layer management of the software and databases including installation, updating and removal is performed. he IT personnel responsible for managing this layer must be well-aware of JavaScript, XML and Perl languages, as well as back-end infrastructure applications like Apache, Tomcat and SQL.

\subsection{Benefits of cloud computing}

\subsubsection{Rapid sales cycle}

With no cost or time to get clients started, and the ability to provide a "try and buy" approach, it is quick and easy to sign up business users.

\subsubsection{Cost of delivery}

A self-service, multi-tenant, cloud solution means vendors can offer their solution equally to major corporations and to the "long tail" of SME customers just as cost effectively. No longer do vendors have to focus on the high margin multinationals, with their associated high cost of sales. 


\subsubsection{Ease of updates}

Unlike on-premise solution, the bug can quickly fix.. This should reduce support calls and ensure an innovation lead over competitors.

\subsubsection{Customer Success and benchmarking}

With the usage patterns of every customer visible to the vendor, the vendor can make suggestions to drive up the benefits the customers are getting which will lead to further sales. But the vendor can also benchmark across customers to help laggards catch up and this can be another valuable revenue stream.

\subsubsection{Annuity revenue model}

The monthly revenue recognition can be painful to start with when cash is king and there is a risk that customers will churn. But in the long term the annuity revenue stream gives a great deal of long-term business confidence.

\section{JOB SCHEDULING ALGORITMS 2.1 FCFS (First Come First Serve)}

It is one of the ancient methods of scheduling in which the job which gets to the scheduler first would be executed first. There are a lot of drawbacks of FCFS ALGORITHM like it does not have any priority concept in it [1].

\subsection{Round Robin Algorithm}

It is the next generation algorithm of FCFS. In this algorithm each job is allocated with a time frame and jobs gets executed to the time frame only.

\subsection{Priority Algorithm}

In this algorithm, each and every job is assigned to a priority and the job gets executed according to the priority assigned to them [5]

\subsection{Time based Scheduling}

In this algorithm the jobs are distributed according to the time frame assigned to the jobs. Increasing time or decreasing time algorithm may be one of the examples of time based scheduling [6].

\section{RESEARCH METHODOLOGY}

The research done in this paper is mainly focused on decreasing the computational cost of the job scheduling. There are mainly three sections in this research work.

\subsection{Task Generation System}

As real time scenario may incorporate any type of tasks hence this research work is consisted of task generation system in which tasks can be generated according to the real time scenario. The parameters over which the tasks are generated are as follows

\subsubsection{Processor Requirement}

It defines the total processor requirement of a job, and if the requirement of the jobs would be satisfied by the system only then it would be transferred to that system else the scheduler would look for another system

\subsubsection{Energy Dissipation Ratio}

It is the amount of energy which a job would consume whiles its execution. This is a major factor over which the grouping would have to be done.

\subsection{Server Generation System}

As to execute the jobs, a scheduler requires system on which the execution can be done we have kept a server generation system in which system would be designed.

\subsection{Design of the Scheduler}

The scheduler design is one of the major part of this research work.

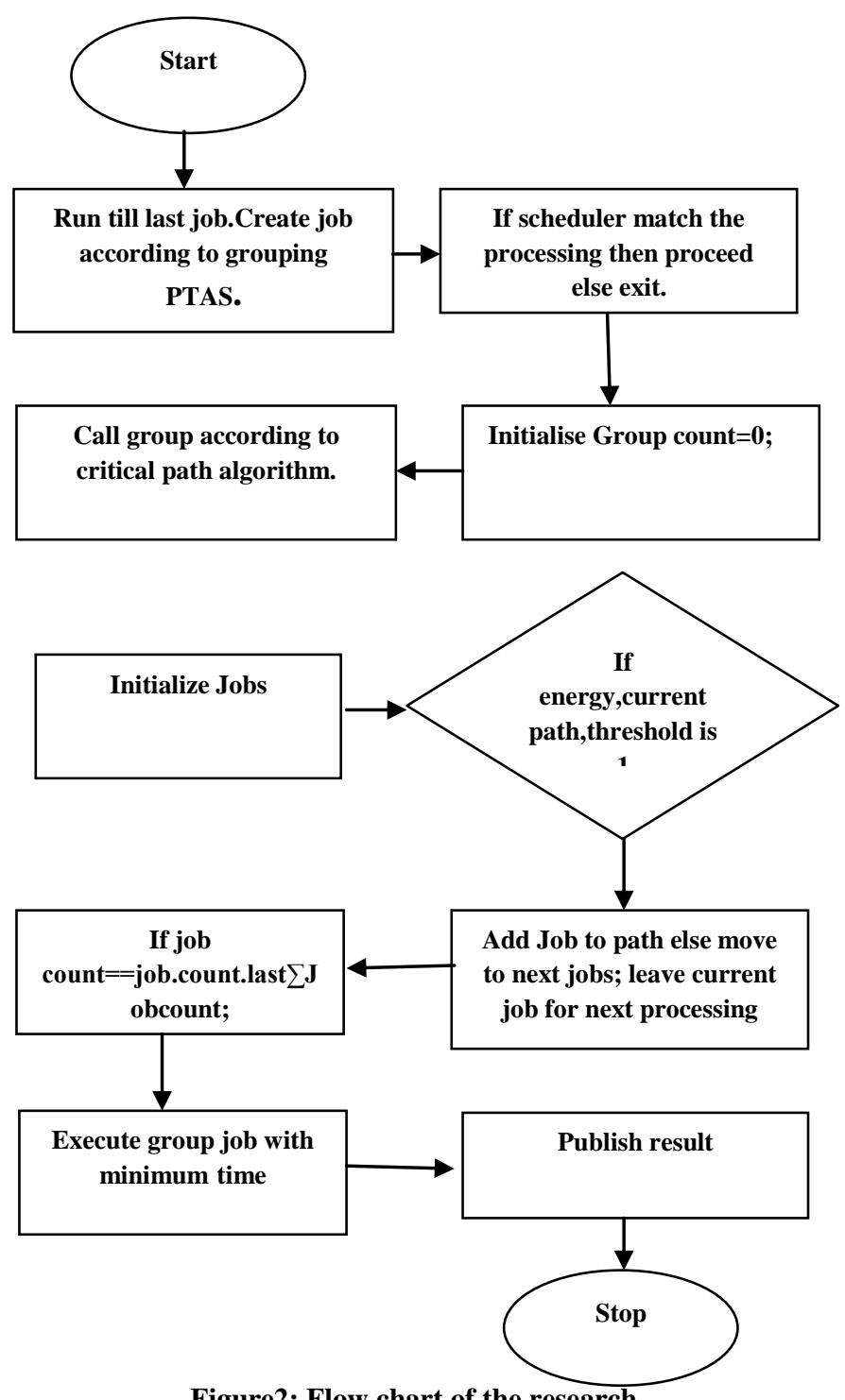

Figure2: Flow chart of the research

Flow charts that showing working of hybrid algorithm, which is a combination of two different algorithms critical path algorithm and decreasing time algorithm. There are some steps that perform during the execution are as:

(i) Start is the first step that initializes the process in the system. It is calling the next step of the hybrid algorithm that is under the loop statement.

(ii) Here system checks the all selected jobs that are use to execute on scheduler. It create the groups of these jobs for execution and for loop that is use for run that code for the number of jobs that are selected. And control goes to next step. 
(iii) Now the algorithm checks the jobs requirements with the system specification to allocate it with the group in system execution. If the specifications are not matched with it the control goes to another system for execution.

(iv) The loop statement that perform the operation up to the job groups. Control goes to next step.

(v) v. Now this step critical path algorithm use to check the energy efficient system path for execution of the job groups.

(vi) Here if the energy used more than required then it execute the else statement for select the another path to execute it.

(vii) Now we have all system and task groups to execute on the scheduler. Here the decreasing time algorithm perform their function to optimize the execution pattern of jobs. It checks the minimum time for execution the jobs and make a pattern to execute.

(viii)The list of jobs that ready to execute on scheduler.

(ix) The scheduler executes all jobs and gives the result to the output screen.

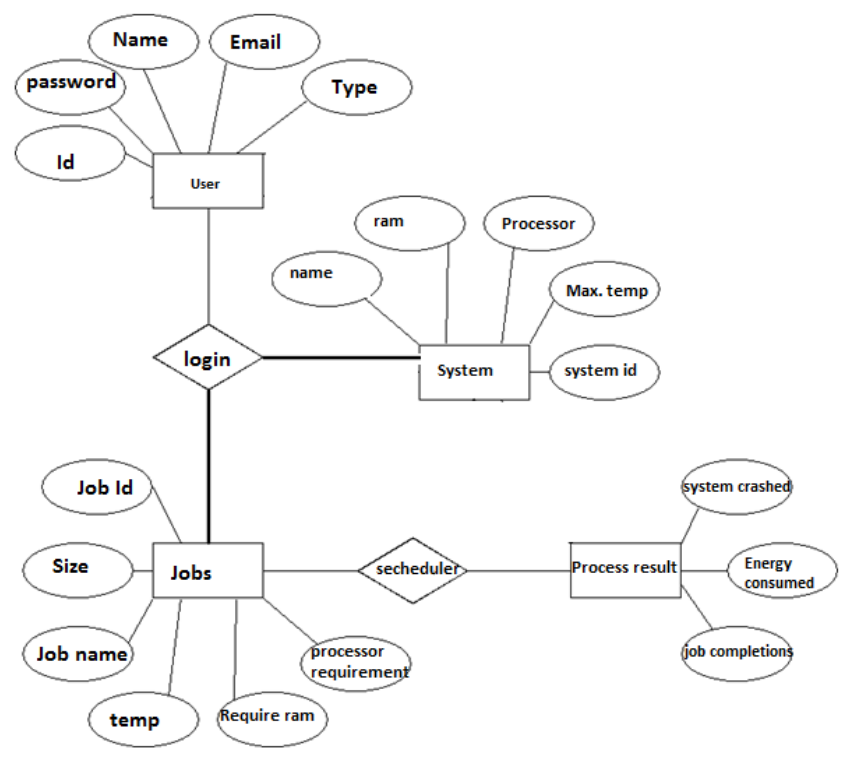

Figure 3: ER diagram of the system

\section{RESULTS AND CONCLUSIONS}

On the basis of the algorithm, the following results are produced. In a given interval of time of 100 mille seconds the cross breed algorithm performs most efficiently where as the FCFS is found to be least efficient. The current approach signifies its importance in the job scheduling environment although the working ability of this algorithm is still to be tested on multiprocessor environment.

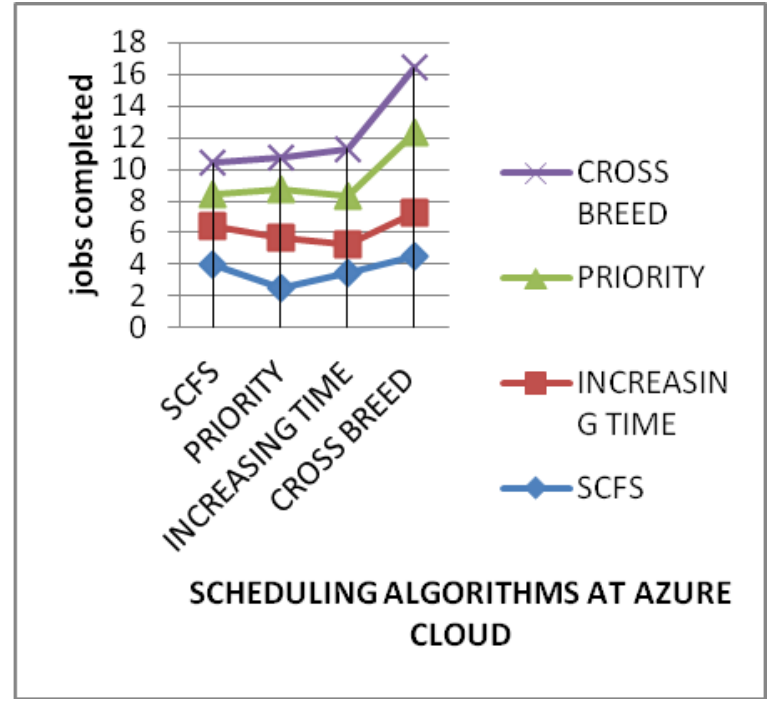

Fig.4: Results Produced

\section{REFERENCES}

[1] Shahram Behzad, "Queue based Job Scheduling algorithm for CloudComputing", International Research Journal of Applied and Basic Sciences (C) ISSN 2251838X / Vol, 4 (12): 3785-3790 Science Explorer Publications.

[2] Pinky Rosemarry, Ravinder Singh, "Grouping Based Job Scheduling Algorithm Using Priority Queue And Hybrid Algorithm In Grid Computing," International Journal of Grid Computing \& Applications (IJGCA) Vol.3, No.4, December 2012.

[3] Jasbir Singh1 and Gurvinder Singh,"Task Scheduling using Performance Effective Genetic Algorithm for Parallel Heterogeneous System", International Journal of Computer Science and Telecommunications [Volume 3, Issue 3, March 2012].

[4] Philippe Baptiste , Marek Chrobak , and Christoph D"urr, "Polynomial Time Algorithms for Minimum Energy Scheduling", IEEE 2010.

[5] Neetu Goel, "A Comparative Study of CPU Scheduling Algorithms," IJGIP Journal homepage: www.ifrsa.org.

[6] Jagbeer Singh, "An Algorithm to Reduce the Time Complexity of Earliest Deadline First Scheduling Algorithm in Real-Time System", (IJACSA) International Journal of Advanced Computer Science and Applications, Vol. 2, No.2, February 2011.

[7] Sotomayor, b., rubã $\odot$, a., llorente, i. And foster, i. 2009. Virtual Infrastructure Management in Private and Hybrid Clouds. Internet Computing, IEEE 13, 5, 14-22.

[8] MEI, L., Chan, W. K. and TSE, T. H. 2008. A Tale of Clouds: Paradigm Comparisons and Some Thoughts on Research Issues. In Asia-Pacific Services Computing Conference, 2008. APSCC '08. IEEE, 464-469.

[9] Lai, C-F., Chang, J-H., Hu, C-C., Huang, Y-M., \& Chao, H-C., (2011). CPRS: A cloud-based program recommendation system for digital TV platforms. Journal Future Generation Computer Systems, Vol. 27, Issue 6. Retrieved October 25, 2011 from http://dl.acm.org/citation.cfm?id=1967928. 
[10] Manvi, S.S., \& Venkataram, P., (2004). Applications of agent technology in communications: a review. Computer Communications. Vol. 27, Issue 15, Pages: 1493-1508. Retrieved October 25, 2011 from http://www.sciencedirect.com/science/article/pii/S01403 66404001914
[11] NIST Definition of Cloud Computing v15, csrc.nist.gov/groups/ SNS/cloud-computing/cloud-defv15.doc.

[12] Cloud Hosting, CLoud Computing and Hybrid Infrastructure from GoGrid, http://www.gogrid.com. 